\title{
Tapentadol in the management of chronic low back pain: a novel approach to a complex condition?
}

This article was published in the following Dove Press journal:

Journal of Pain Research

20 July $201 \mathrm{I}$

Number of times this article has been viewed

\author{
Joseph Pergolizzi' \\ Eli Alon ${ }^{2}$ \\ Ralf Baron ${ }^{3}$ \\ Cesare Bonezzi ${ }^{4}$ \\ Jan Dobrogowski ${ }^{5}$ \\ Rafael Gálvez ${ }^{6}$ \\ Troels Jensen ${ }^{7}$ \\ Hans-Georg Kress ${ }^{8}$ \\ Marco AE Marcus9 \\ Bart Morlion ${ }^{10}$ \\ Serge Perrot ${ }^{\prime \prime}$ \\ Rolf-Detlef Treede ${ }^{12}$ \\ 'Department of Medicine, Johns \\ Hopkins University School of \\ Medicine, Baltimore, MD, USA; \\ ${ }^{2}$ Universitätsspital Zurich, Switzerland; \\ ${ }^{3}$ Universitätsklinikum Schleswig- \\ Holstein, Kiel, Germany; ${ }^{4}$ Fondazione \\ Salvatore Maugeri, Pavia, Italy; ${ }^{5}$ Zakład \\ Badania i Leczenia Bólu, Kraków, \\ Poland; ${ }^{6} \mathrm{Hospital}$ Universitario \\ Virgen de las Nieves, Granada, \\ Spain; ${ }^{7}$ Aarhus University, Denmark; \\ ${ }^{8}$ Medical University of Vienna, \\ Austria; ' Maastricht University \\ Medical Center and University \\ of Muenster, Maastricht, The \\ Netherlands; ${ }^{10}$ University Hospitals \\ Leuven, Belgium; "Hôpital Dieu, Paris, \\ France; ${ }^{12}$ Ruprecht-Karls-University, \\ Heidelberg, Germany
}

Correspondence: Joseph Pergolizzi

Naples Anesthesia and Pain Associates,

4840 Sycamore Drive, Naples, FL 34 I I 9 ,

USA

Tel +I 2395973564

$\mathrm{Fax}+\mathrm{I} 2395977566$

Email jpjmd@msn.com
Abstract: Chronic pain affects approximately 1 in 5 people in Europe, and around half of sufferers receive inadequate pain management. The most common location is the lower back. Pharmacological treatment of this condition is challenging because of the range of causative mechanisms and the difficulty of balancing analgesic efficacy and tolerability. An international panel of clinical pain specialists met in September, 2009, to discuss the treatment of chronic low back pain, and to review preclinical and clinical data relating to the new analgesic, tapentadol. A lack of consensus exists on the best treatment for low back pain. The range of regularly prescribed pharmacological agents extends from nonopioids (paracetamol, NSAIDs, and COX-2 inhibitors) to opioids, antidepressants and anticonvulsants. Pain relief may be compromised, however, by an undetected neuropathic component or intolerable side effects. Treatment is potentially life-long and effective analgesics are urgently needed, with demonstrable long-term safety. Combining separate agents with different mechanisms of action could overcome the limitations of present pharmacological therapy, but clinical evidence for this approach is currently lacking. Tapentadol combines $\mu$-opioid agonism with noradrenaline reuptake inhibition in a single molecule. There is strong evidence of synergistic antinociception between these two mechanisms of action. In preclinical and clinical testing, tapentadol has shown efficacy against both nociceptive and neuropathic pain. Preclinical data indicate that tapentadol's $\mu$-opioid agonism makes a greater contribution to analgesia in acute pain, while noradrenaline reuptake inhibition makes a greater contribution in chronic neuropathic pain models. Tapentadol also produces fewer adverse events than oxycodone at equianalgesic doses, and thus may have a ' $\mu$-sparing effect'. Current evidence indicates that tapentadol's efficacy/ tolerability ratio may be better than those of classical opioids. However, further research is needed to establish its role in pain management.

Keywords: chronic low back pain, neurophysiological changes, neuropathic component, multimechanistic approach, efficacy/side effect ratio, tapentadol

\section{Introduction}

Chronic pain represents a major healthcare problem which seriously impairs the quality of sufferers' working and social lives, and the most common single location is the lower back. ${ }^{1}$ Chronic low back pain (LBP) is difficult to treat because of the range of causative mechanisms that may be involved; this difficulty is compounded by the limited efficacy of current pharmacological agents, and exemplified by the inconsistency of existing treatment patterns. Moreover, optimal pain management demands not only effective pain relief, but a precise balance of analgesia, tolerability, and beneficial effect on functionality/quality of life. 
In September 2009, an international panel of clinical pain specialists met to discuss the challenges of chronic LBP, and to review the preclinical and clinical data relating to the new analgesic agent, tapentadol, which may offer a novel treatment option for chronic LBP. Based on a literature search of PubMed before the meeting, 20 unique articles were chosen by the moderator for presentation to the panel. Other presentations came from articles relating to the changes occurring in chronic LBP and the preclinical and clinical testing of tapentadol, as well as data on file from completed clinical trials.

\section{Prevalence of low back pain}

A 2006 survey of 46,394 adults in 15 European countries and Israel found that $19 \%$ of respondents suffered from moderate to severe chronic pain ( $\geq 5$ on a 10-point Numeric Rating Scale [NRS]) and nearly half received inadequate pain management. ${ }^{1}$ Other surveys in Norway ${ }^{2}$ and Denmark ${ }^{3}$ have produced similar results. The economic implications for society are significant in terms of the utilization of healthcare resources. ${ }^{4}$

In the pan-European study, the most common location of chronic pain was the back, in $47 \%$ of sufferers ( $5 \%$ upper back, $18 \%$ lower back, $24 \%$ unspecified). ${ }^{1}$ This finding is consistent with other sources of prevalence data on low back pain, indicating an overall prevalence of 80.5 per 1000 population. ${ }^{5}$ It affects more women than men and the prevalence increases with age. ${ }^{5,6}$ In the US, LBP accounts for almost 30 million physician visits per year- $56 \%$ of which are to primary care physicians. ${ }^{7}$ Thus LBP imposes a significant economic burden. In Sweden alone, the cost of LBP in 2001 was estimated to be $€ 1860$ million, ${ }^{8}$ while in the US the overall cost of back pain is estimated to exceed US\$15 billion per year. ${ }^{9}$

\section{Complexity of treating low back pain}

LBP is defined as pain located in the lumbosacral region of the spine, the most frequent location being the fourth and fifth lumbar segment. It may be classified according to its etiology; mechanical or nonspecific LBP has no serious underlying pathology or nerve root compromise, in contrast to secondary LBP. ${ }^{10}$ It may also be classified according to its duration into 1 of 3 phases: acute, subacute (intermittent), or chronic. Definitions vary, but LBP is generally regarded as chronic if symptoms persist for more than 3 to 6 months. ${ }^{11}$ The transition to chronic LBP involves structural and neurophysiological changes, such as peripheral and central sensitization. ${ }^{12}$ At this point the patient's symptoms may be exacerbated by psychosocial factors such as irritability, emotional distress, social withdrawal, excessive consumption of alcohol or medication, and loss of income. ${ }^{13}$ The result may be persistent disability, depression, or anxiety. The impact on quality of life is considerable. ${ }^{14}$ Early diagnosis is therefore important, to incorporate these aspects of the condition into the management of low back pain and increase the chance of recovery. ${ }^{15}$

\section{Discussion}

Despite the substantial economic burden of chronic LBP, there is a lack of consensus on the best treatment. ${ }^{16}$ Patients have very different levels of impairment, disability, and chronicity. Cases of low impairment and disability may best be treated by simple evidence-based therapies - exercises, cognitive-behavioral interventions, and medication - but the multimechanistic nature of more severe chronic LBP means that no single intervention is likely to be effective. ${ }^{12,17}$

The complexity of treatment is further increased because chronic LBP may present with nociceptive pain, neuropathic pain, or both components..$^{18,19}$ One survey using the painDETECT questionnaire found that $37 \%$ of chronic LBP patients suffered from neuropathic pain, while $35 \%$ had nociceptive pain and results for the remaining patients were unclear. ${ }^{20}$ A neuropathic component can complicate diagnosis for several reasons. ${ }^{21}$ Firstly, the signs and symptoms can vary between patients and within individual patients over time. The clinical picture may be obscured by psychosocial factors, which need to be addressed. This is important because neuropathic pain often fails to respond to commonly prescribed analgesic therapy, ${ }^{21}$ treatment with single agent drugs being limited by incomplete efficacy and dose-limiting adverse effects. ${ }^{22}$

The major pharmacological agents currently used onlabel to treat chronic LBP include nonopioids, such as paracetamol, nonsteroidal anti-inflammatory drugs (NSAIDs), cyclooxygenase-2 (COX-2) inhibitors, and opioids, while antidepressants, anticonvulsants, and local anesthetics may also be prescribed. However, any benefit can be adversely affected by several factors. For example, efficacy may be limited by an undetected neuropathic component, and poor tolerability may lead to an unfavorable efficacy/side effect ratio. Opioid-related side effects, in particular, affect $73 \%$ to $90 \%$ of patients treated for longer than 3 months and are directly responsible for high withdrawal rates of $20 \%$ to $40 \%{ }^{23}$ Treatment may also be compromised by drug-drug interactions or the development of tolerance, demonstrating the urgent need for analgesics which are effective and safe in long-term use. 
These limitations can be addressed by combining agents with different mechanisms of action, to produce additive or synergistic analgesic effects and minimize drug-induced adverse events. ${ }^{24}$ This multimechanistic approach takes into account the diversity of underlying pain mechanisms and the different pharmacological principles required for their successful treatment. However, such polypharmacy also presents challenges. It reduces the convenience of treatment, and thus compliance, and creates the potential for pharmacokinetic drug-drug interactions. ${ }^{25}$ These may increase side effects or reduce analgesia, and the elderly, in particular, may be at increased risk. ${ }^{25}$ Therefore the safety and efficacy of specific combinations must be empirically evaluated, ${ }^{22}$ but up to now clinical study data are inadequate. An alternative multimechanistic approach is to develop novel compounds which possess multiple mechanisms of action.

\section{The pharmacology of tapentadol}

Tapentadol (see Table 1) has been developed to have broad analgesic activity by combining known analgesic principles ( $\mu$-opioid receptor agonism and noradrenaline reuptake inhibition) in a single molecule, and to offer a better balance between efficacy and tolerability than classical opioids. An immediate release formulation has recently been registered in the US to treat moderate to severe acute pain, and both immediate and prolonged formulations are currently undergoing registration in Europe for the treatment of severe acute and chronic pain.

Tapentadol's agonistic activity at the $\mu$-opioid receptors of afferent pain fibres inhibits the release of excitatory neurotransmitters, and reduces the upward transmission of pain signals. Via its $\mu$-opioid action in the brain, tapentadol also influences the release of neurotransmitters by the descending pain pathways, producing a further inhibition of pain. ${ }^{26}$ Like other systemic opioids, tapentadol acts at all these sites and its overall analgesic effect is enhanced by synergy between them. ${ }^{27}$

Opioids can be effective in chronic neuropathic pain, but decreased potency requires higher doses in order to achieve sufficient analgesia. ${ }^{28,29}$ This characteristic of neuropathic pain,

Table I Properties of tapentadol

\begin{tabular}{ll}
\hline Chemical formula & $\mathrm{C}_{14} \mathrm{H}_{23} \mathrm{NO} \cdot \mathrm{HCl}$ \\
Molecular weight & 257.80 \\
Racemic mixture & No (single enantiomer) \\
Primary metabolic pathway & Phase II glucuronidation \\
Active metabolites & No \\
Mechanism of action & $\mu$-opioid receptor agonist/ \\
& noradrenaline reuptake inhibitor \\
\hline
\end{tabular}

and the development of tolerance, both potentially increase the incidence of side effects. Tapentadol addresses this problem by virtue of its second analgesic mechanism of action, ie, noradrenaline reuptake inhibition, particularly in chronic neuropathic pain. This property has been reported by studies in a number of animal models. ${ }^{30}$ Noradrenaline reuptake inhibition exerts its antinociceptive effect via the descending pain pathways, where the increased synaptic noradrenaline binds to $\alpha-2$ receptors and thereby reduces pain signals to the brain. ${ }^{31}$ There is strong evidence of synergistic antinociception between this mechanism of action and $\mu$-opioid agonism. ${ }^{32-35}$

Other analgesic agents also possess more than 1 mechanism of action; for example, at first sight the established weak opioid tramadol appears similar to tapentadol, since it is a $\mu$-opioid receptor agonist which also affects the monoaminergic system. However, there are important differences. Both tapentadol's mechanisms of action reside in a single molecule that is metabolized via $O$-glucuronidation to an inactive metabolite. ${ }^{36}$ By contrast, tramadol is a racemic mixture of 2 enantiomers and produces an active metabolite. While the (-)-enantiomer and (+)-enantiomer of the parent compound produce noradrenaline reuptake inhibition and serotonin reuptake inhibition, respectively, weak $\mu$-opioid receptor agonism resides mainly in the (+)-enantiomer of $O$-desmethyl-tramadol, the major active metabolite. Thus, the relative contribution of the different mechanisms of action to the overall analgesic effect changes over time. ${ }^{36}$ As the parent molecule is metabolised, noradrenaline reuptake inhibition and serotonin reuptake inhibition diminish, and $\mu$-opioid receptor agonism increases. This produces a complex timeand metabolism-dependent pattern of pharmacological activities. ${ }^{36}$ By contrast, tapentadol does not rely on metabolic activation to achieve full $\mu$-opioid receptor agonism. Also, tramadol is metabolized mainly by the cytochrome P450 system, which is polymorphic in humans, so that 'poor metabolizers' do not experience satisfactory analgesia with standard doses. ${ }^{36}$ Further differences favoring tapentadol over tramadol are that evidence suggests that analgesia is more readily obtained by noradrenaline reuptake inhibition than by serotonin reuptake inhibition..$^{36,37}$

\section{Tapentadol: preclinical testing}

Despite having an affinity to the $\mu$-opioid receptor 50 times lower than that of morphine, in vivo testing in rats and mice found that intravenous tapentadol was only 2 to 3 times less potent than morphine in acute nociceptive pain. ${ }^{36,37}$ In 2 animal models of chronic neuropathic pain it was broadly equipotent to morphine, despite this much lower binding affinity, which is 
important in terms of opioid side effects. ${ }^{36}$ Thus noradrenaline reuptake inhibition may make a greater contribution to the analgesic efficacy of tapentadol in chronic pain than in acute pain. This hypothesis is supported by a study which combined tapentadol with either the $\mu$-opioid antagonist naloxone or the $\alpha-2$ antagonist yohimbine. ${ }^{30}$ In Figure 1, the dose-response curve shifted much further to the right when the $\mu$-opioid action of tapentadol was antagonised in acute pain. In chronic pain, however, the reverse applied; antagonizing noradrenaline reuptake inhibition had the greater effect. ${ }^{30}$

Low affinity for the $\mu$-opioid receptor and the presence of noradrenaline reuptake inhibition suggest that tapentadol might produce fewer opioid-related side effects than classical $\mu$-opioid receptor agonists. The emetic potential of tapentadol and morphine has been compared in ferrets; tapentadol produced fewer retches and vomits per animal, and the duration of these effects was shorter. ${ }^{37}$ In mice, tapentadol had a weaker inhibitory effect than morphine on gastrointestinal motility. ${ }^{37}$ When the development of tolerance was investigated in rats, complete tolerance was significantly delayed $(P<0.0001)$ in animals receiving tapentadol (23 days) compared with those receiving morphine (10 days). ${ }^{36}$

To summarise, tapentadol's $\mu$-opioid action decreased ascending pain messages as well as increasing pain inhibition via the descending pathways. Simultaneous blocking of the noradrenaline transporter further enhanced its analgesic effects via the increased activation of $\alpha-2$ receptors. Thus there is a possible synergy between the mechanisms of action and also the sites of action. Because its distinct pharmacological profile differentiates tapentadol from other centrally acting analgesics, it has been proposed that tapentadol should be classified by its 2 mechanisms of action, $\mu$-opioid receptor agonism (MOR) and noradrenaline reuptake inhibition (NRI), as a MOR-NRI compound. ${ }^{38}$

\section{Tapentadol: clinical trials in chronic LBP}

Tapentadol has been subject to an extensive testing program; to date, around 8000 patients have participated in Phase III clinical trials with either the immediate-release (IR) or prolonged-release (PR) formulation. Chronic LBP was 1 major pain model used in the phase III clinical program and has been included in the current phase IIIB program, to gather further evidence on the use of tapentadol in this prevalent chronic pain condition.

To evaluate the efficacy and safety of multiple doses of tapentadol PR, 981 patients with chronic LBP were recruited to a double-blind, randomized, active- and placebo-controlled, phase III study. ${ }^{39}$ A 3-week titration phase allowed patients to achieve their optimal individual dose of tapentadol PR (100-250 mg twice a day), oxycodone controlled release (CR; 20-50 mg twice a day), or placebo. During the following 12-week maintenance phase, patients were not permitted rescue medication but were allowed to adjust their dosage, to reflect clinical practice. The primary endpoint was the mean change in pain intensity at week 12 or over the entire 12-week period, using the last observation carried forward (LOCF) imputation.

Demographic and baseline characteristics were consistent across treatment groups, the majority of subjects being women and below 65 years of age. ${ }^{39}$ Severe pain $(\mathrm{NRS} \geq 6)$ was reported by $88.5 \%$ of subjects and $53.4 \%$ had prior opioid experience.
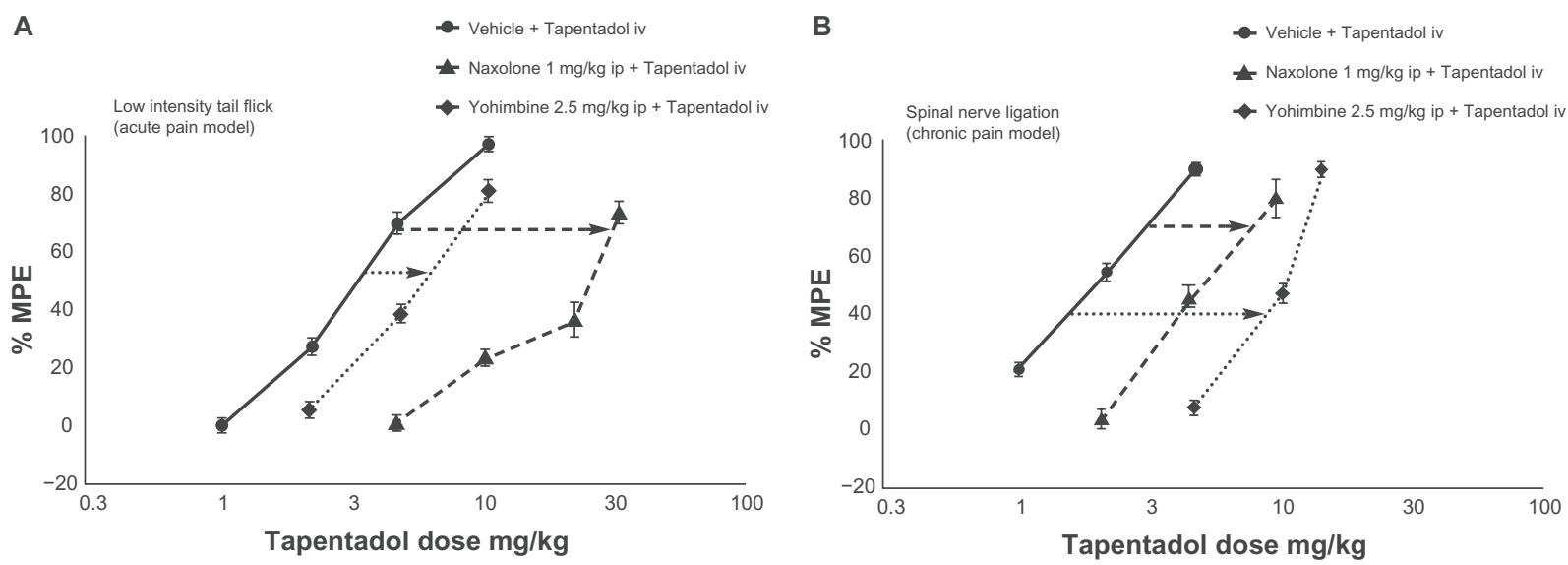

Figure I Differential contribution of $\mu$-opioid agonism and noradrenaline reuptake inhibition in acute and chronic neuropathic pain models. ${ }^{30}$ In acute pain, antagonizing $\mu$-opioid agonism with naloxone moves the dose-response curve further to the right than antagonizing noradrenaline reuptake inhibition with yohimbine, showing that $\mu$-opioid agonism makes a greater contribution to the compound's analgesic effect. In chronic neuropathic pain, the opposite is true; noradrenaline reuptake inhibition contributes more to analgesia.

Reprinted from European Journal of Pain, vol 14, issue 8. Schröder W, De Vry J, Tzschentke TM, Jahnel U, Christoph T. Differential contribution of opioid and noradrenergic mechanisms to the antinociceptive and antihypersensitive efficacy of tapentadol in rat models of nociceptive and neuropathic pain, 8I4-82I, Copyright (20I0), with permission from Elsevier.

Abbreviation: MPE, maximum possible effect. 


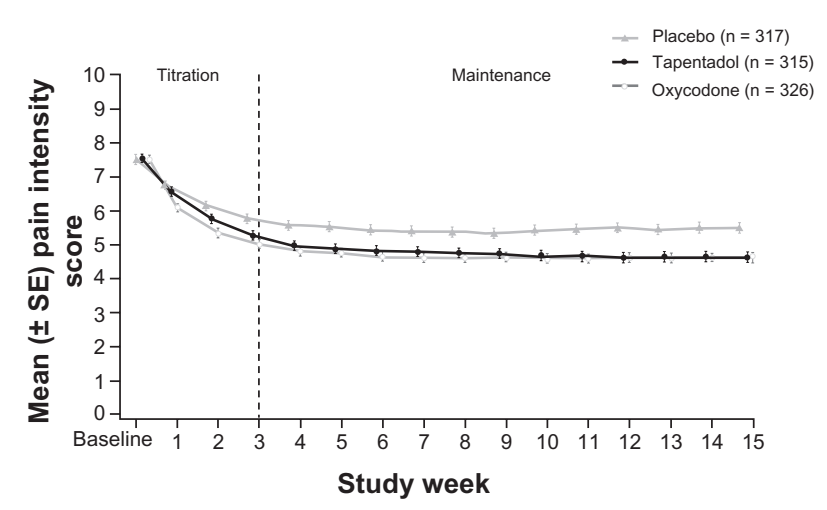

Figure 2 Average pain intensity scores over time..$^{39}$ Pain relief was consistent during the maintenance period for both active treatment groups. Tapentadol PR significantly reduced mean pain intensity compared with placebo at week 12 and throughout the maintenance period, using the last observation carried forward imputation method for missing values.

Used with permission of Informa Healthcare, from Expert Opinion on Pharmacotherapy, Buynak R, et al, Vol I I, Issue I I, 20 I 0; permission conveyed through Copyright Clearance Center, Inc.

Abbreviations: $\mathrm{CR}$, controlled release; PR, prolonged release.

As can be seen from Figure 2, both active treatment groups produced a comparable, statistically significant, reduction in pain intensity over the maintenance period (both $P<0.001$ vs placebo). ${ }^{39}$ Reductions were similar in opioid-naïve and opioid-experienced subjects. The results for tapentadol were supported by most secondary outcome parameters and also by more conservative imputations, such as worst observation carried forward (WOCF) and baseline observation carried forward (BOCF). ${ }^{39}$ Tapentadol also performed significantly better than placebo in all categories of the Brief Pain Inventory, in overall sleep quality ratings and in the Patients' Global Impression of Change (PGIC). ${ }^{39}$

Tapentadol was well tolerated, the incidence of typical opioid adverse events being approximately half that of oxycodone ${ }^{39}$ At dosages providing similar analgesic effects, tapentadol produced numerically lower levels of constipation ( $13.8 \%$ vs $26.8 \%)$, nausea $(20.1 \%$ vs $34.5 \%)$, vomiting $(9.1 \%$ vs $19.2 \%)$, dizziness ( $11.9 \%$ vs $17.1 \%)$ and pruritis $(7.2 \%$ vs $16.8 \%) .{ }^{39} \mathrm{CNS}$ effects tended to be milder in the tapentadol group than the oxycodone group..$^{39}$ These tolerability findings are consistent with tapentadol having a ' $\mu$-sparing effect', owing to the contribution of the NRI component.

Figure 3 shows the percentage of patients in the different treatment groups who discontinued treatment over the study period. The main cause of discontinuation in the active treatment groups was treatment-emergent adverse events, predominantly gastrointestinal side effects. Discontinuations for this reason were lower in the tapentadol group than the oxycodone group, the disparity being particularly marked during the titration phase. ${ }^{39}$

The efficacy of tapentadol in treating purely neuropathic pain has been investigated in 395 patients suffering from

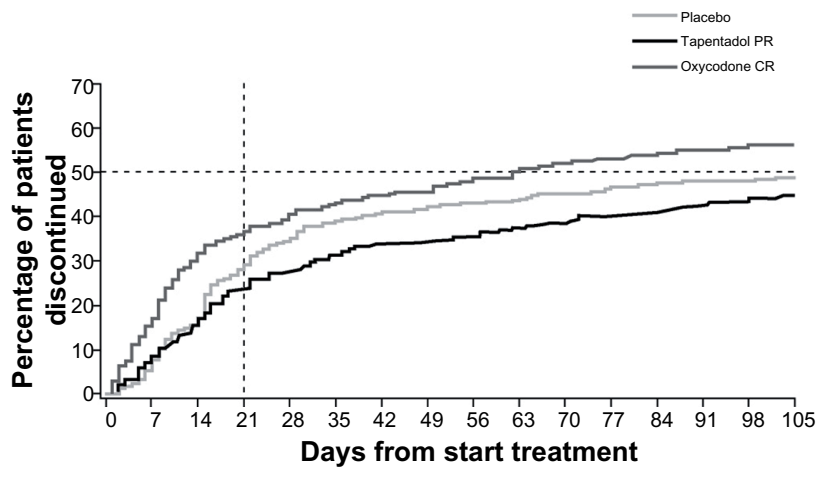

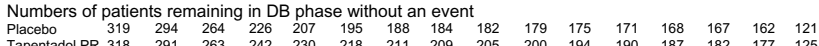

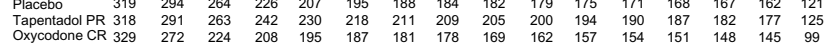

Figure 3 Treatment discontinuations over time. ${ }^{39}$ The time to discontinuation in the tapentadol PR group was significantly longer than in the oxycodone CR group $(P<0.00 \mathrm{I})$, but not significantly different from that in the placebo group $(P=0.309)$. The primary reason for treatment discontinuation in the active treatment groups was adverse events (16.7\% of the tapentadol PR group, compared with $32.3 \%$ of the oxycodone CR group).

Used with permission of Informa Healthcare, from Expert Opinion on Pharmacotherapy, Buynak R, et al, Vol I I, Issue I I, 20 I0; permission conveyed through Copyright Clearance Center, Inc.

Abbreviations: CR, controlled release; PR, prolonged release.

painful diabetic neuropathy. ${ }^{40}$ After a 3-week open-label titration phase, subjects who responded to tapentadol were assigned in a 1:1 ratio to receive either tapentadol PR or placebo for a 12-week, fixed-dose, double-blind phase. Rescue medication was allowed during this phase, and the doses of tapentadol spanned the entire therapeutic range, from $100 \mathrm{mg}$ twice a day to $250 \mathrm{mg}$ twice a day. The primary endpoint was to show a statistically significant difference in pain intensity between tapentadol and placebo at week 12, using an 11-point NRS and the LOCF imputation. This was achieved $(P<0.001)$ (see Figure 4) and efficacy was confirmed

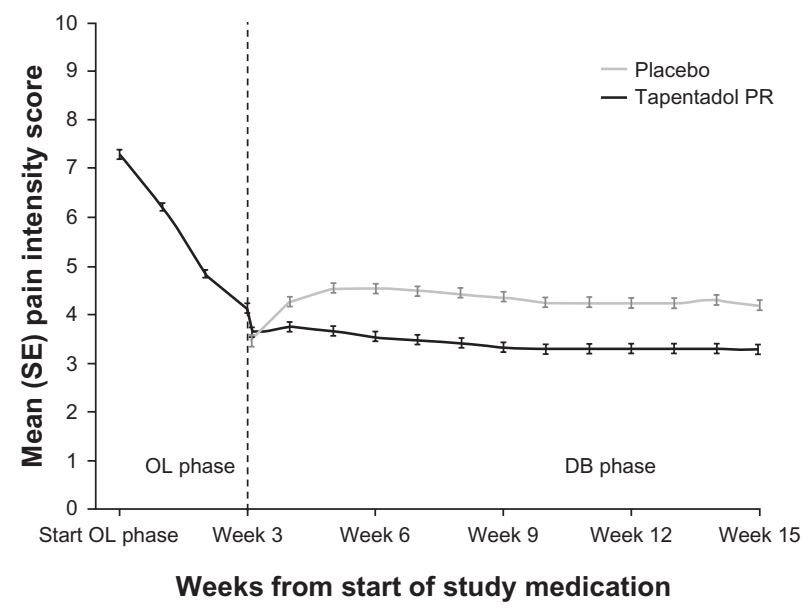

Figure 4 Weekly average pain intensity scores. ${ }^{40}$ During the double-blind maintenance period, the mean pain intensity increased in the placebo group but remained stable in the tapentadol PR group. Tapentadol PR also showed a favorable tolerability profile, with a maximum of about $20 \%$ for individual adverse events. Used with permission of Informa Healthcare, from Current Medical Research and Opinion, Schwartz S, et al, Vol 27, Issue I, 20I I; permission conveyed through Copyright Clearance Center, Inc.

Abbreviations: OL, open label; DB, double blind; PR, prolonged release. 
A

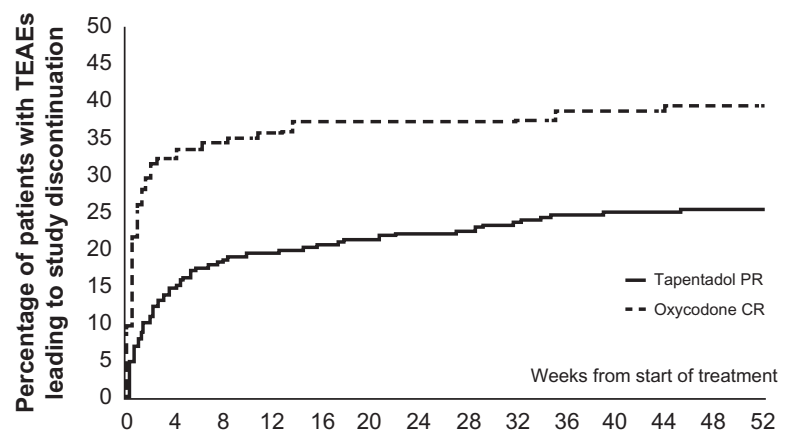

B

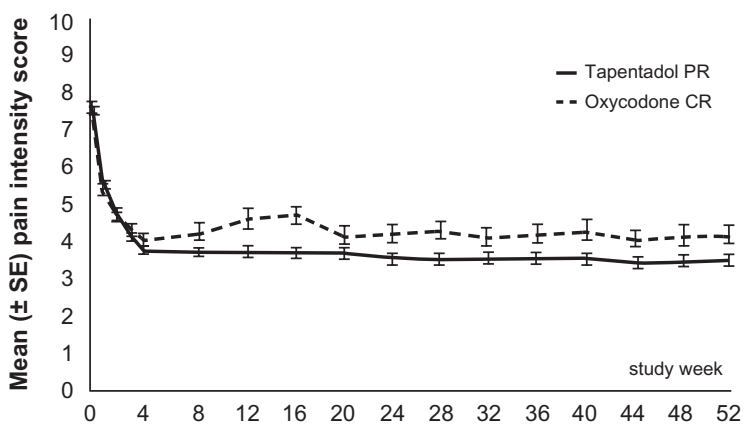

Figure 5 Discontinuations and mean pain intensity scores over time in patients suffering from low back pain and osteoarthritis. 41,42 A) The percentage of patients who discontinued treatment because of adverse events was lower for tapentadol than for oxycodone (22.7\% vs $36.8 \%)$. The overall percentage of patients who discontinued treatment was also lower for tapentadol than for oxycodone (53.8\% vs 65\%). B) Tapentadol PR provided sustainable relief of moderate to severe chronic knee or hip osteoarthritis or low back pain for up to I year. The efficacy of tapentadol PR was comparable to that of oxycodone CR.

Figure 5B from Pain Practice, Vol 10, Wild et al. Copyright (C) 2010 by John Wiley \& Sons, Inc. Reprinted by permission of John Wiley \& Sons, Inc; permission conveyed through Copyright Clearance Center, Inc.

Abbreviations: CR, controlled release; PR, prolonged release; TEAEs, treatment-emergent adverse events.

by more conservative analyses of the primary endpoint, including BOCF, WOCF, and the proportions of patients showing $\geq 30 \%$ and $\geq 50 \%$ improvement. ${ }^{40}$

Long-term safety has been evaluated in a Phase III, open-label, randomized, active-controlled study ${ }^{41} \mathrm{~A}$ total of 1117 patients with chronic LBP or osteoarthritis were randomised (4:1) to receive tapentadol PR or oxycodone CR. A 1-week titration phase enabled subjects to determine their optimal dose of analgesic, within the range of 100 to $250 \mathrm{mg}$ twice a day for tapentadol PR, or 20 to $50 \mathrm{mg}$ twice a day for oxycodone CR. This was followed by a 51-week maintenance phase, during which patients were encouraged to stay on a stable dose, but could adjust it if necessary, and were allowed paracetamol rescue medication. The primary endpoint was to determine the safety of tapentadol over 1 year. ${ }^{41}$

The majority of subjects were women and under 65 years of age. Around two-thirds suffered from chronic LBP. At baseline, $89.4 \%$ had severe pain and the median pain intensity was 8.0 on an 11-point NRS. Approximately half the patients were opioid-naïve.

The mean dose of tapentadol for those who completed the study remained unchanged over the 1 -year period. ${ }^{41}$ Figure 5B shows that the mean pain intensity also remained unchanged, indicating that tapentadol produced a consistent reduction in pain over the total study period. There were fewer gastrointestinal and CNS adverse events in patients receiving tapentadol than in those receiving oxycodone. ${ }^{42}$ Fewer patients in the tapentadol group withdrew from treatment because of adverse events (Figure 5A), and fewer patients in the tapentadol group discontinued treatment for any reason..$^{42}$ In this study, therefore, the tolerability of tapentadol was superior to that of oxycodone over a period of 1 year.

In these clinical trials, tapentadol provided reliable analgesia in chronic LBP, comparable to that of oxycodone, a representative traditional opioid. It was effective against both nociceptive and neuropathic pain. At equianalgesic doses, tapentadol produced a lower incidence of side effects than oxycodone, leading to fewer patients withdrawing from treatment. Clinical studies in patients with acute and chronic osteoarthritis pain, and with acute postoperative pain, have produced similar results. ${ }^{41,43,44}$ Further clinical trials are currently under way comparing tapentadol with other analgesics.

\section{Conclusion}

The treatment of chronic LBP is challenging for various reasons, and requires a multidisciplinary approach to treatment, encompassing pharmacological, psychological, and physical therapies. The efficacy of classical opioids can be compromised by dose restrictions as a result of side effects or the development of tolerance. Tapentadol combines $\mu$-opioid receptor agonism and noradrenaline reuptake inhibition in a single molecule, with the object of achieving an 'opioid-sparing' effect, that is, to produce fewer opioidrelated side effects for a given level of analgesia.

To date, preclinical and clinical data support the pharmacological classification of tapentadol as an MOR-NRI ${ }^{30,38}$ and suggest that its efficacy/tolerability ratio may be better than those of classical opioids..$^{36,37,39,41}$ Further research is required, however, particularly into efficacy and safety in patients with pure neuropathic pain and to provide specific data on LBP with a neuropathic component. Comparisons 
are also required between tapentadol and classical opioids other than oxycodone.

\section{Acknowledgment}

This article was based on a meeting held in Brussels, Belgium, on September 4 and 5, 2009, and was supported by Grünenthal GmbH, Aachen, Germany.

\section{Disclosure}

The following authors received honoraria to attend the meeting in Brussels: Joseph Pergolizzi, Cesare Bonezzi, Jan Dobrogowski, Rafael Gálvez, Troels S. Jensen, Hans-Georg Kress, Marco A. E. Marcus, Bart Morlion, Serge Perrot, Rolf-Detlef Treede.

The authors thank Derrick Garwood Ltd, Cambridge, UK, for editorial support, which was sponsored by Grünenthal $\mathrm{GmbH}$, Aachen, Germany.

\section{References}

1. Breivik H, Collett B, Ventafridda V, Cohen R, Gallacher D. Survey of chronic pain in Europe: prevalence, impact on daily life, and treatment. Eur J Pain. 2006;10:287-333.

2. Rustoen T, Wahl AK, Hanestad BR, Lerdal A, Paul S, Miaskowski C. Prevalence and characteristics of chronic pain in the general Norwegian population. Eur J Pain. 2004;8:555-565.

3. Sjøgren P, Ekholm O, Peuckmann V, Grønbaek M. Epidemiology of chronic pain in Denmark: an update. Eur J Pain. 2009;13: 287-292.

4. Annemans L. Pharmacoeconomic impact of adverse events of longterm opioid treatment for the management of persistent pain. Clin Drug Investig. 2011;31:73-86.

5. Andersson GB. Epidemiological features of chronic low-back pain. Lancet. 1999;354:581-585.

6. Mapel DW, Shainline M, Paez K, Gunter M. Hospital, pharmacy, and outpatient costs for osteoarthritis and chronic back pain. J Rheumatol. 2004;31:573-583.

7. Hart LG, Deyo RA, Cherkin DC. Physician office visits for low back pain: frequency, clinical evaluation, and treatment patterns from a US national survey. Spine. 1995;20:11-19.

8. Ekman M, Johnell O, Lidgren L. The economic cost of low back pain in Sweden in 2001. Acta Orthop. 2005;76:275-284.

9. Stewart WF, Ricci JA, Chee E, Morganstein D, Lipton R. Lost productive time and cost due to common pain conditions in the US workforce. JAMA. 2003;290:2443-2454.

10. Kuritzky L, Barnett A, Doan H, et al. The 10-minute examination for low back pain. J Musculoskelet Med. 2002;19:497-505.

11. Turk DC, Okifuji A. Pain terms and taxonomies of pain. In: Loeser JD, Butler SH, Chapman CR, Turk DC, editors. Bonica's Management of Pain, 3rd ed. Baltimore, Maryland: Lippincott Williams \& Wilkins; 2001: $17-25$.

12. Raffa RB, Pergolizzi JV, Tallarida RJ. The determination and application of fixed-dose analgesic combinations for treating multimodal pain J Pain. 2010;11:701-709.

13. Livengood JM. Psychologic and psychosocial factors contributing to chronic pain. Curr Rev Pain. 1999;3:1-9.

14. Lame IE, Peters ML, Vlaeyen JW, Kleef M, Patijin J. Quality of life in chronic pain is more associated with beliefs about pain, than with pain intensity. Eur J Pain. 2005;9:15-24.

15. Van Tulder M, Koes B, Bombardier C. Low back pain. Best Pract Res Clin Rheumatol. 2002;16:761-775.
16. Pai S, Sundaram LJ. Low back pain: an economic assessment in the United States. Orthop Clin North Am. 2004;35:1-5.

17. Backpain Europe. European Guidelines For the Management of Chronic Non-Specific Low Back Pain (Amended Version). http:// www.backpaineurope.org/web/files/WG2_Guidelines.pdf. Accessed September 28, 2009.

18. Portenoy RK, Kanner RM. Definition and assessment of pain. In: Portenoy RK, Kanner RM, editors. Pain Management Theory and Practice. Philadelphia, PA: FA Davis Co; 1996:3-18.

19. Galer BS, Dworkin RH. A Clinical Guide to Neuropathic Pain. Minneapolis, Minnesota: The McGraw-Hill Companies Inc; 2000.

20. Freynhagen R, Baron R, Gockel U, Tölle TR. painDETECT: a new screening questionnaire to identify neuropathic components in patients with back pain. Curr Med Res Opin. 2006;22: 1911-1920.

21. Harden N, Cohen M. Unmet needs in the management of neuropathic pain. J Pain Symptom Manage. 2003;25(Suppl 5):S12-S17.

22. Gilron I, Max MB. Combination pharmacotherapy for neuropathic pain: current evidence and future directions. Expert Rev Neurotherapeutics. $2005 ; 5: 823-830$

23. Martell BA, O'Connor PG, Kerns RD, et al. Systematic review: opioid treatment for chronic back pain: prevalence, efficacy, and association with addiction. Ann Intern Med. 2007;146:116-127.

24. Hartrick CT. Multimodal postoperative pain management. Am J Health Syst Pharm. 2004;61(Suppl 1):S4-S10.

25. Shakib S. Problems of polypharmacy. Aust Fam Physician. 2002;31: $125-128$.

26. Pasternak GW. Molecular insights into mu opioid pharmacology: from the clinic to the bench. Clin J Pain. 2010;26(Suppl 10): S3-S9.

27. Niv D, Nemirovsky A, Rudick V, Geller E, Urca G. Antinociception induced by simultaneous intrathecal and intraperitoneal administration of low doses of morphine. Anesth Analg. 1995;80:886-889.

28. Ballantyne JC, Mao JM. Opioid therapy for chronic pain. NEngl J Med. 2003;349:1943-1953.

29. Joo DT. Mechanisms of opioid tolerance: emerging evidence and therapeutic implications. Can J Anaesth. 2007;54:969-976.

30. Schröder W, De Vry J, Tzschentke TM, Jahnel U, Christoph T. Differential contribution of opioid and noradrenergic mechanisms of tapentadol in rat models of nociceptive and neuropathic pain. Eur J Pain. 2010; 14;814-821.

31. Rahman W, D'Mello R, Dickenson AH. Peripheral nerve injury-induced changes in spinal $\alpha_{2}$-adrenoceptor-mediated modulation of mechanically evoked dorsal horn neuronal responses. J Pain. 2008;9:350-359.

32. Garrido MJ, Valle M, Campanero MA, Calvo R, Trocóniz IF. Modeling of the in vivo antinociceptive interaction between an opioid agonist, (+)-O-desmethyltramadol, and a monoamine reuptake inhibitor, (-)-O-desmethyltramadol, in rats. J Pharmacol Exp Ther. 2000;295:352-359.

33. Ossipov MH, Suarez LJ, Spaulding TC. Antinociceptive interactions between alpha 2-adrenergic and opiate agonists at the spinal level in rodents. Anesth Analg. 1989;68:194-200.

34. Sullivan AF, Kalso EA, McQuay HJ, Dickenson AH. Evidence for the involvement of the mu but not the delta opioid receptor subtype in the synergistic interaction between opioid and alpha 2 adrenergic antinociception in the rat spinal cord. Neurosci Lett. 1992;139: 65-68.

35. Schröder W, Tzschentke TM, Terlinden R, et al. Synergistic interaction between the two mechanisms of action of tapentadol in analgesia J Pharmacol Exp Ther. 2011;337:312-320.

36. Tzschentke TM, Christoph T, Kögel B, et al. (-)-(1R,2R)-3(3-dimethylamino-1-ethyl-2-methyl-propyl)-phenol hydrochloride (tapentadol $\mathrm{HCl}$ ): a novel mu-opioid receptor agonist/norepinephrine reuptake inhibitor with broad-spectrum analgesic properties. $J$ Pharmacol Exp Ther. 2007;323:265-276.

37. Tzschentke TM, De Vry J, Terlinden R, et al. Tapentadol hydrochloride. Drugs Fut. 2006;31:1053-1061. 
38. Kress H. (Editorial) Tapentadol and its two mechanisms of action: Is there a new pharmacological class of centrally acting analgesics on the horizon? Eur J Pain. 2010;14:781-783.

39. Buynak R, Shapiro DY, Okamoto A, et al. Efficacy and safety of tapentadol extended release for the management of chronic low back pain: results of a prospective, randomized, double-blind, placeboand active-controlled Phase III study. Expert Opin Pharmacother. 2010;11:1787-1804.

40. Etropolski MS, Shapiro DY, Okamoto A, Rauschkolb C, Häeussler J. Safety and tolerability of tapentadol extended release in patients with painful diabetic peripheral neuropathy: Results of a randomizedwithdrawal phase 3 study. Diabetes. 2009;58:A226-A227. Abstract 852-P.

41. Wild JE, Grond S, Kuperwasser B, et al. Long-term safety and tolerability of tapentadol extended release for the management of chronic low back pain or osteoarthritis pain. Pain Pract. 2010;10:416-427.
42. Weber H, Lange R, Kuperwasser B, et al. Tolerability of tapentadol prolonged release based on discontinuations due to adverse events in a 1-Year Randomized Phase 3 Safety Study. Poster presented at the 6th Triennial Congress of the European Federation of Chapters of the International Association for the Study of Pain (EFIC), Lisbon, Portugal; 9-12 September, 2009.

43. Lange B, Kuperwasser B, Okamoto A, et al. Efficacy and safety of tapentadol prolonged release for chronic osteoarthritis pain and low back pain. Adv Ther. 2010;27:381-399.

44. Hartrick C. Tapentadol immediate release for the relief of moderate-tosevere acute pain. Expert Opin Pharmacother. 2009;10:2687-2696.

\section{Publish your work in this journal}

The Journal of Pain Research is an international, peer-reviewed, open access, online journal that welcomes laboratory and clinical findings in the fields of pain research and the prevention and management of pain. Original research, reviews, symposium reports, hypothesis formation and commentaries are all considered for publication.

\section{Dovepress}

The manuscript management system is completely online and includes a very quick and fair peer-review system, which is all easy to use. Visit http://www.dovepress.com/testimonials.php to read real quotes from published authors. 